\title{
INFLUÊNCIA DA IDADE E DO NÚMERO DE CICLOS ESTRAIS PRÉVIOS À PRIMEIRA INSEMINAÇÃO NA EFICIÊNCIA REPRODUTIVA DE MATRIZES SUÍNAS
}

\author{
INFLUENCE OF THE NUMBER OF PREVIOUS ESTROUS CYCLES AND AGE AT FIRST \\ INSEMINATION IN REPRODUCTIVE EFFICIENCY OF SOWS
}

\author{
A. C. F. RODRIGUES ${ }^{1}$, E. P. $\operatorname{COSTA}^{1 *}$, V. R. A. MENDES ${ }^{1}$, A. H. A. $\operatorname{COSTA}^{2}$
}

\begin{abstract}
RESUMO
O presente estudo investigou a influência da idade ao primeiro estro e do número de ciclos prévios à primeira cobertura (a partir do segundo) no desempenho reprodutivo de matrizes no primeiro parto. Foram avaliadas 2.710 fêmeas da linhagem Camborough ${ }^{\circledR}$ 25, nascidas entre 2007 e 2012, em sete granjas comerciais situadas na região de Ponte Nova MG. Os dados foram obtidos a partir de bancos de dados dos programas de gerenciamento Pigchamp ${ }^{\circledR}$ e Agriness ${ }^{\circledR}$. O tamanho da primeira leitegada não foi influenciado $(\mathrm{P}>0,05)$ pela idade à primeira inseminação, idade ao primeiro estro $(\mathrm{P}>0,05)$ e pelo número de estros $(\mathrm{P}>0,05)$ em que a marrã foi inseminada a primeira vez, nas sete granjas. A taxa de repetição de estros também não foi influenciada $(\mathrm{p}>0,05)$ pela idade à primeira inseminação. Pode-se concluir para a linhagem avaliada, que a taxa de repetição de estro e o tamanho da leitegada no primeiro parto não são influenciados pela idade ao primeiro estro, assim como pela idade e número de estros à primeira inseminação. Diante destas considerações, as marrãs devem ser inseminadas a partir do segundo estro, visando um menor período de dias não produtivos, sem comprometimento da eficiência reprodutiva.
\end{abstract}

PALAVRAS-CHAVE: Estro. Leitegada. Marrãs. Reprodução. Suinocultura

\section{SUMMARY}

The present study investigated the influence of age and the numbers of estrus at first mating (from the second) and age at first estrus on reproductive performance of sows at first farrowing. Gilts Camborough ${ }^{\circledR} 25$ lineage were evaluated, born between 2007 and 2012, from seven commercial farms situated in Ponte Nova - MG. Data were obtained from databases of management programs PigChamp ${ }^{\circledR}$ and Agriness ${ }^{\circledR}$. The size of the first litter was not influenced $(\mathrm{P}>0.05)$ by age at first insemination, age at first estrus $(\mathrm{P}>0,05)$ and by the number of estrus in which the gilts were inseminated the first time $(\mathrm{P}>0,05)$, in the seven farms. The repetition rate of estrus also was not influenced by age at first insemination $(\mathrm{P}>0,05)$. It is conclude for lineage evaluated, that the repetition rate of estrus and the litter size at first farrowing are not influenced by age at first estrus, as well as by age and number of estrus at first insemination. In view of these considerations, gilts should be inseminated as from the second estrus, aiming a smaller period of nonproductive days, without commitment of reproductive efficiency.

KEY-WORDS: Estrus. Litter. Gilts. Reproduction. Pig farming

\footnotetext{
${ }^{1}$ Universidade Federal de Viçosa

${ }^{2}$ Médica Veterinária Autônoma

*Autor para correspondência: epcosta@ufv.br
} 


\section{INTRODUÇÃO}

O Brasil é o $4^{\circ}$ maior produtor mundial de carne suína, contabilizando 39,3 milhões de cabeças em 2011. Neste mesmo ano, foram abatidos 34,9 milhões de suínos, sendo Minas Gerais responsável por 11,8\% deste total (ABIPECS, 2012). Índices de reposição médios de até $50 \%$ fazem com que mais de um milhão de marrãs ingressem no rebanho brasileiro anualmente, demandando, em muitas unidades de produção, equipes especializadas no manejo desta categoria de animais. Este tem sido um ponto importante, exigindo cada vez mais a atenção e dedicação dos produtores e técnicos a esta categoria de fêmeas.

Em função destas elevadas taxas de reposição aplicadas na suinocultura moderna, a eficiência reprodutiva da marrã assume papel de destaque. Este grupo de animais representa um percentual considerável nos custos gerais da produção, pois, constituem cerca de 30 á $40 \%$ do plantel de fêmeas na maioria dos rebanhos. Diante disto, é indispensável o correto manejo com a leitoa de reposição, pois os fatores que afetam sua vida útil reprodutiva podem ter influência significante no desempenho do rebanho como um todo (KIRKWOOD \& AHERNE, 1985).

A produtividade da fêmea suína está associada ao número de leitões nascidos vivos no primeiro parto. Assim, quanto maior a prolificidade das marrãs no primeiro parto, melhores serão seus resultados nos partos subsequentes e, consequentemente, estas fêmeas terão um melhor desempenho reprodutivo e econômico durante toda sua vida produtiva (EDWARDS, 1997; MARTIN RILLO et al., 2000).

Uma das bases para manter uma alta prolificidade é determinar o momento ideal da cobertura das nulíparas, permitindo um correto desenvolvimento dos órgãos genitais, essencial para uma adequada eficiência reprodutiva, particularmente na primeira gestação e parto. $\mathrm{O}$ efeito do tamanho da leitegada no primeiro parto sobre a produtividade média da porca durante toda a sua vida tem sido demonstrado em diferentes estudos. De acordo com as pesquisas realizadas nesta área, o número de nascidos vivos no primeiro parto influencia a produtividade da porca nos partos sucessivos (MARTIN RILLO et al., 2000).

$\mathrm{O}$ manejo adequado das marrãs é um aspecto fundamental para um bom desempenho ao longo de sua vida reprodutiva. Fatores como a nutrição, o período de adaptação e a imunização contra agentes específicos podem ser limitantes para o desempenho reprodutivo nos partos subsequentes. Além disso, a idade, o peso, o número de estros antes da inseminação artificial (IA) e os cuidados durante a gestação (alojamento) e a primeira lactação podem também influenciar os índices reprodutivos de uma matriz (KIRKWOOD \& AHERNE, 1985).

Neste contexto, a antecipação da puberdade e da primeira inseminação são aspectos importantes, visto que as leitoas são responsáveis pelo maior número de dias não produtivos (DNP) do plantel (LUCIA Jr et al., 2000). O principal objetivo das investigações ligadas às novas biotécnicas aplicadas no manejo reprodutivo deve ser a adequação das novas linhagens a precocidade ao primeiro estro, à puberdade e a primeira concepção, associadas às condições preconizadas de idade e peso corporal.

O objetivo deste trabalho foi verificar, por meio de uma análise retrospectiva, o efeito da idade ao primeiro estro e da idade e número de estros à primeira cobertura no desempenho reprodutivo de matrizes no primeiro parto.

\section{MATERIAL E MÉTODOS}

O presente experimento foi realizado por meio da análise de dados obtidos a partir de bancos dos programas de gerenciamento de granjas Pigchamp ${ }^{\circledR}$ e Agriness ${ }^{\circledR}$. As planilhas de dados foram complementadas e conferidas por meio da análise das fichas individuais das matrizes, tendo em vista que nem todas as informações necessárias estavam digitadas nos programas de gerenciamento.

Foram avaliadas 2.710 matrizes da linhagem Camborough $^{\circledR} 25$, tendo em vista ser uma linhagem mais recente em uso no mercado e, ao mesmo tempo, já conter dados suficientes relacionados à eficiência reprodutiva das fêmeas. Este procedimento foi adotado tendo em vista que, ao longo dos anos, as empresas de genética retiram do mercado a linhagem atual, a partir do momento em que disponibilizam uma nova. Destarte, é de fundamental importância que sejam avaliadas linhagens mais atuais, tendo em vista que as mais antigas não estão mais disponíveis pelas empresas de genética ou não mais existem nas granjas.

Foram avaliados dados de sete granjas situadas na região de Ponte Nova - MG. De cada marrã foi compilado o histórico relacionado à idade do animal ao primeiro estro, à primeira inseminação, ao número de estros apresentados antes da primeira inseminação e resultados relacionados ao parto. $\mathrm{O}$ número de repetições utilizado para cada variável estudada variou em função dos dados disponíveis nas fichas das matrizes. Quando o número de repetições foi menor ou igual a 20, os dados não foram tabulados ou analisados dados, tendo em vista a instabilidade da variável quantitativa descontínua "tamanho da leitegada".

Nas sete granjas, as marrãs foram alojadas em baias coletivas, com cerca de seis a oito animais por baia e com uma densidade de $1,80 \mathrm{~m}^{2}$ por animal. Os galpões de pré e pós-cobertura eram equipados com ventiladores e aqueles com uma maior incidência solar, protegidos por árvores ou sombrites. Para estimular a puberdade, o contato com o macho foi iniciado nas fêmeas partir de 150 dias de idade. O macho foi colocado duas vezes ao dia nas baias das leitoas, por cerca de 10 a 15 minutos. A detecção de estro foi realizada por um funcionário treinado. Foram realizadas três inseminações por estro observado, com intervalos de 12 horas cada, sendo a primeira no momento da detecção do estro.

A ração para as leitoas foi fornecida duas vezes ao dia contendo $18 \%$ de proteína bruta, cerca de $0,9 \%$ de lisina e $3.100 \mathrm{Kcal} / \mathrm{Kg}$ de energia metabolizável. Em torno de 10 dias antes da primeira inseminação as marrãs 
foram movidas para gaiolas com fornecimento de ração à vontade durante todo o dia. Durante a gestação, a composição da ração fornecida nas granjas continha $18 \%$ de proteína bruta, $0,7 \%$ de lisina e $3.050 \mathrm{Kcal} / \mathrm{Kg}$ de energia metabolizável. Foram fornecidos $2 \mathrm{~kg}$ desta ração até 85 dias de gestação e $3 \mathrm{~kg}$ de 86 até o parto. Durante a lactação, a ração foi composta por $19 \%$ de proteína bruta, $1,25 \%$ de lisina e $3.450 \mathrm{Kcal} / \mathrm{Kg}$ de energia metabolizável fornecida à vontade. Em todas as etapas a água foi fornecida à vontade durante todo o dia.

As variáveis estudadas foram comparadas em dois períodos definidos em função da idade ao primeiro estro e da primeira inseminação. Assim, foram comparados os dados de 2.657 marrãs que apresentaram o primeiro estro até 190 dias com aquelas com mais de 190 dias de idade. Também foram comparados parâmetros reprodutivos de 2.710 marrãs inseminadas com 190 a 220 dias de idade com as inseminadas após este período. O período avaliado foi diferente daquele definido para o primeiro estro (até 190 e após 190 dias), tendo em vista o reduzido número de inseminações realizadas até 190 dias de idade. As análises foram realizadas individualmente para cada granja.

As variáveis quantitativas foram submetidas aos testes de Normalidade (Lilliefors) e Homocedasticidade (Cochran) e posteriormente a análise de variância. As médias foram comparadas utilizando o teste $\mathrm{F}$, adotando-se o nível de $5 \%$ de probabilidade. Quando não atendiam as premissas de normalidade e homocedasticidade, mesmo após as transformações apropriadas, os dados foram submetidos ao teste não-paramétrico de Wilcoxon (SAEG, 1999).

As variáveis qualitativas foram comparadas em tabelas de contingência e analisadas pelo teste de quiquadrado a 5\% de probabilidade (SAMPAIO, 2002).

\section{RESULTADOS E DISCUSSAO}

\section{Idade ao primeiro estro (IDPE) e desempenho reprodutivo no primeiro parto}

Não foram observadas diferenças $(P>0,05)$ no tamanho médio da leitegada, dos animais que apresentaram o primeiro estro até 190 dias, quando comparado com fêmeas que apresentaram este estro após os 190 dias de idade (Tabela 1). Estes resultados corroboram com os valores observados por Brooks \& Smith (1980), Patterson et al. (2010) e Roongsitthichai et al. (2013), no qual não registraram diferença $(\mathrm{P}>0,05)$ no número de leitões nascidos vivos e nascidos

totais.

Tabela 1 - Efeito da idade ao primeiro estro (IDPE) no tamanho da leitegada do primeiro parto.

\begin{tabular}{|c|c|c|c|c|c|c|}
\hline Granja & Tratamento & $\mathrm{N}$ & $\overline{\mathrm{MM}}$ & NM & $\mathrm{NV}$ & NT \\
\hline A & $\begin{array}{l}\text { IDPE1 } \\
\text { IDPE2 }\end{array}$ & $\begin{array}{c}1.025 \\
237\end{array}$ & $\begin{array}{cc}\mathrm{m} & \mathrm{s} \\
0,28 \pm 0,73 \\
0,27 \pm 0,63\end{array}$ & $\begin{array}{cc}\mathrm{m} & \mathrm{s} \\
0,43 & \pm 0,81 \\
0,45 & \pm 0,85\end{array}$ & $\begin{array}{cc}\mathrm{m} & \mathrm{s} \\
10,68 \pm 3,07 \\
10,99 \pm 2,85\end{array}$ & $\begin{array}{c}\mathrm{m} \\
11,39 \pm 3,06 \\
11,72 \pm 2,89\end{array}$ \\
\hline B & $\begin{array}{l}\text { IDPE1 } \\
\text { IDPE2 }\end{array}$ & $\begin{array}{l}518 \\
140\end{array}$ & $\begin{array}{l}0,28 \pm 0,75 \\
0,33 \pm 0,82\end{array}$ & $\begin{array}{l}0,53 \pm 1,13 \\
0,51 \pm 0,72\end{array}$ & $\begin{array}{l}10,35 \pm 3,08 \\
10,64 \pm 2,98\end{array}$ & $\begin{array}{l}11,16 \pm 3,05 \\
11,48 \pm 3,18\end{array}$ \\
\hline $\mathrm{C}$ & $\begin{array}{l}\text { IDPE1 } \\
\text { IDPE2 }\end{array}$ & $\begin{array}{l}71 \\
17\end{array}$ & $\begin{array}{l}0,27 \pm 0,94 \\
0,35 \pm 0,70\end{array}$ & $\begin{array}{l}0,35 \pm 0,93 \\
0,41 \pm 1,06\end{array}$ & $\begin{array}{l}10,41 \pm 3,51 \\
10,65 \pm 2,26\end{array}$ & $\begin{array}{l}11,03 \pm 3,27 \\
11,41 \pm 2,26\end{array}$ \\
\hline D & $\begin{array}{l}\text { IDPE1 } \\
\text { IDPE2 }\end{array}$ & $\begin{array}{c}147 \\
31\end{array}$ & $\begin{array}{l}0,07 \pm 0,35 \\
0,19 \pm 0,40\end{array}$ & $\begin{array}{l}0,26 \pm 0,59 \\
0,16 \pm 0,37\end{array}$ & $\begin{array}{l}11,33 \pm 2,85 \\
11,84 \pm 3,16\end{array}$ & $\begin{array}{l}11,67 \pm 2,89 \\
12,19 \pm 3,46\end{array}$ \\
\hline $\mathrm{E}$ & $\begin{array}{l}\text { IDPE1 } \\
\text { IDPE2 }\end{array}$ & $\begin{array}{l}88 \\
57\end{array}$ & $\begin{array}{l}0,25 \pm 0,57 \\
0,35 \pm 0,74\end{array}$ & $\begin{array}{l}0,43 \pm 0,75 \\
0,58 \pm 0,86\end{array}$ & $\begin{array}{l}11,69 \pm 2,35 \\
11,68 \pm 2,77\end{array}$ & $\begin{array}{l}12,37 \pm 2,47 \\
12,61 \pm 2,97\end{array}$ \\
\hline $\mathrm{F}$ & $\begin{array}{l}\text { IDPE1 } \\
\text { IDPE2 }\end{array}$ & $\begin{array}{c}195 \\
48\end{array}$ & $\begin{array}{l}0,30 \pm 0,59 \\
0,19 \pm 0,44\end{array}$ & $\begin{array}{l}0,42 \pm 0,75 \\
0,46 \pm 1,15\end{array}$ & $\begin{array}{l}10,44 \pm 2,94 \\
10,17 \pm 2,85\end{array}$ & $\begin{array}{l}11,16 \pm 3,26 \\
10,81 \pm 3,08\end{array}$ \\
\hline G & $\begin{array}{l}\text { IDPE1 } \\
\text { IDPE2 }\end{array}$ & $\begin{array}{l}58 \\
25\end{array}$ & $\begin{array}{l}0,29 \pm 0,56 \\
0,40 \pm 0,71\end{array}$ & $\begin{array}{l}0,36 \pm 0,58 \\
0,36 \pm 0,49\end{array}$ & $\begin{array}{l}12,10 \pm 2,41 \\
10,96 \pm 2,82\end{array}$ & $\begin{array}{l}12,79 \pm 2,64 \\
11,64 \pm 2,96\end{array}$ \\
\hline
\end{tabular}

Não houve diferença entre tratamentos na mesma granja $(\mathrm{P}>0,05)$ pelo teste $\mathrm{F}$, quanto ao tamanho da leitegada.

N: número de repetições; MM: mumificados; NM: natimortos; NV: nascidos vivos; NT: nascidos totais; m: média; s: desvio padrão. IDPE 1: idade ao primeiro estro até 190 dias; IDPE 2: idade ao primeiro estro após 190 dias.

No presente estudo, as médias das idades ao primeiro estro de cada granja ocorreram entre $170 \mathrm{e}$ 190 dias, semelhante à média verificada por Karlbom et al. (1982). Diante disto, é provável que estas fêmeas tenham atingido suficiente desenvolvimento corporal para manifestar o primeiro estro até 190 dias.

Quanto às fêmeas que apresentaram o primeiro estro mais tardiamente (mais do que 190 dias de idade), essas poderão apresentar um comprometimento da produtividade ao longo de sua vida reprodutiva. Este fato se deve ao aumento dos dias não produtivos na granja e a diminuição do total de partos durante a sua vida útil (PATERSON, 1982; SCHUKKEN et al., 1994). Entretanto, no presente estudo, o tamanho da leitegada ao primeiro parto das fêmeas que apresentaram o primeiro estro após 190 dias não foi comprometido. 


\section{Idade à primeira inseminação (IDPI) e desempenho reprodutivo no primeiro parto}

No presente estudo, não foram observadas diferenças $(\mathrm{P}>0,05)$ no tamanho das leitegadas em marrãs inseminadas com até 220 dias de idade, quando comparadas com as inseminadas após os 220 dias, dentro de cada granja. Da mesma forma, a taxa de repetições de estro foi muito reduzida, demonstrando que não foi influenciada $(\mathrm{P}>0,05)$ pela idade à primeira inseminação (Tabela 2).

Tabela 2 - Efeito da idade à primeira inseminação (IDPI) no tamanho da leitegada do primeiro parto e no número de repetições de estro

\begin{tabular}{|c|c|c|c|c|c|c|c|}
\hline Granja & $\mathrm{N}$ & Tratamento & MM & NM & $\mathrm{NV}$ & NT & $\begin{array}{l}\text { Repetição de } \\
\text { estro }\end{array}$ \\
\hline \multirow{3}{*}{ A } & & & $\mathrm{m} \quad \mathrm{s}$ & $\mathrm{m} \quad \mathrm{s}$ & $\mathrm{m} \quad \mathrm{s}$ & $\mathrm{m} \quad \mathrm{s}$ & \\
\hline & 508 & IDPI 1 & $0,26 \pm 0,71$ & $0,41 \pm 0,80$ & $10,91 \pm 2,96$ & $11,58 \pm 3,01$ & $17(3,3 \%)$ \\
\hline & 793 & IDPI 2 & $0,29 \pm 0,72$ & $0,44 \pm 0,82$ & $10,63 \pm 3,08$ & $11,36 \pm 3,04$ & $27(3,4 \%)$ \\
\hline \multirow{2}{*}{ B } & 103 & IDPI 1 & $0,20 \pm 0,55$ & $0,56 \pm 0,87$ & $10,04 \pm 3,19$ & $10,80 \pm 3,40$ & $2(1,9 \%)$ \\
\hline & 559 & IDPI 2 & $0,30 \pm 0,79$ & $0,51 \pm 1,09$ & $10,47 \pm 3,03$ & $11,29 \pm 3,01$ & $7(1,3 \%)$ \\
\hline \multirow{2}{*}{$\mathrm{C}$} & 59 & IDPI 1 & $0,27 \pm 0,74$ & $0,44 \pm 1,12$ & $10,22 \pm 3,26$ & $10,93 \pm 3,27$ & $1(1,7 \%)$ \\
\hline & 29 & IDPI 2 & $0,31 \pm 0,17$ & $0,21 \pm 0,41$ & $10,93 \pm 3,37$ & $11,45 \pm 2,72$ & $1(3,4 \%)$ \\
\hline \multirow{2}{*}{ D } & 43 & IDPI 1 & $0,05 \pm 0,21$ & $0,21 \pm 0,56$ & $11,70 \pm 2,51$ & $11,95 \pm 2,60$ & $2(4,7 \%)$ \\
\hline & 135 & IDPI 2 & $0,11 \pm 0,40$ & $0,26 \pm 0,56$ & $11,32 \pm 3,02$ & $11,70 \pm 3,11$ & $5(3,7 \%)$ \\
\hline \multirow[b]{2}{*}{ E } & 83 & IDPI 1 & $0,24 \pm 0,53$ & $0,53 \pm 0,89$ & $11,48 \pm 2,71$ & $12,25 \pm 2,77$ & $0(0,0 \%)$ \\
\hline & 72 & IDPI 2 & $0,32 \pm 0,73$ & $0,49 \pm 0,79$ & $11,83 \pm 2,29$ & $12,64 \pm 2,51$ & $0(0,0 \%)$ \\
\hline \multirow{2}{*}{$\mathrm{F}$} & 24 & IDPI 1 & $0,50 \pm 0,83$ & $0,54 \pm 0,88$ & $10,17 \pm 2,88$ & $11,21 \pm 3,32$ & $0(0,0 \%)$ \\
\hline & 219 & IDPI 2 & $0,25 \pm 0,52$ & $0,41 \pm 0,83$ & $10,41 \pm 2,93$ & $11,08 \pm 3,21$ & $0(0,0 \%)$ \\
\hline \multirow{2}{*}{ G } & 33 & IDPI 1 & $0,27 \pm 0,57$ & $0,27 \pm 0,52$ & $11,61 \pm 2,49$ & $12,15 \pm 2,60$ & $0(0,0 \%)$ \\
\hline & 50 & IDPI 2 & $0,36 \pm 0,63$ & $0,42 \pm 0,57$ & $11,86 \pm 2,66$ & $12,64 \pm 2,89$ & $0(0,0 \%)$ \\
\hline
\end{tabular}

Não houve diferença entre tratamentos na mesma granja $(\mathrm{P}>0,05)$ pelo teste $\mathrm{F}$, quanto ao tamanho da leitegada.

Não houve diferença entre tratamentos na mesma granja $(\mathrm{P}>0,05)$ pelo teste do qui-quadrado, quanto a taxa de repetição de estro. N: número de repetições; MM: mumificados; NM: natimortos; NV: nascidos vivos; NT: nascidos totais; m: média; s: desvio padrão. IDPI 1: Idade à primeira inseminação de 190 a 220 dias; IDPI 2: Idade à primeira inseminação após 220 dias.

Com relação à repetição de estro, Lucia Jr. et al. (2003), observaram resultados diferentes do presente estudo, quanto ao desempenho reprodutivo de leitoas. Os autores concluíram que a idade abaixo de 200 dias está associada com um maior risco de retorno ao estro. Porém, não ocorreram efeitos sobre o tamanho da leitegada e sobre o intervalo desmame-estro após o primeiro parto.

Contrapondo os resultados do presente estudo, Schukken et al. (1994) e Babot et al. (2003) verificaram que, o número de nascidos vivos no primeiro parto foi maior quando a idade à primeira cobertura aumentou. Os autores em questão avaliaram intervalos de idade a primeira inseminação de 200 a 220 dias e 221 a 240 dias. Nos dois trabalhos foi acompanhada a vida reprodutiva das porcas até o abate e foi observado que fêmeas cobertas com idade mais avançada possuem menor expectativa de partos do que aquelas cobertas mais cedo. Esses autores concluíram que, apesar de ter ocorrido um aumento no número de nascidos vivos no primeiro parto quando a idade à primeira cobertura aumentou, deve-se levar em conta a vida reprodutiva da porca como um todo e os custos da granja, e não somente o desempenho do primeiro parto.
Já Saito et al. (2011), avaliando o desempenho de fêmeas cruzadas inseminadas no intervalo de 188 a 365 dias de idade, encontraram um menor número de nascidos vivos no primeiro parto quando as fêmeas foram inseminadas no intervalo de 188 a 229 dias $(\mathrm{P}<0,05)$. Apesar deste menor número de nascidos, essas fêmeas tiveram um maior número de partos até a sua remoção das granjas (0,2 a 0,8 partos a mais) em relação às inseminadas mais tardiamente. Portanto, estes autores não recomendam atrasar a primeira inseminação, visando um melhor desempenho reprodutivo na vida útil.

Nos estudos citados anteriormente (SCHUKKEN et al., 1994; BABOT et al., 2003 e SAITO et al., 2011) os autores agruparam dados de várias granjas, comprometendo a interpretação dos resultados encontrados, tendo em vista que não foram apresentados os resultados de cada granja individualmente.

Assim como o presente estudo, Clark et al. (1988) analisaram a idade de fêmeas mestiças à primeira inseminação em duas granjas individualmente nos intervalos de 180-220, 221-245 e >245. Porém, estes autores verificaram um aumento no tamanho da leitegada quando a idade aumentou de 180 até 245 dias 
$(\mathrm{P}<0,05)$. Este fato pode ser explicado pelo uso de um número maior de animais por granja e um maior número de intervalos de idades analisados, o que provavelmente, tenha favorecido a detecção de diferenças no tamanho da leitegada entre as idades. Diferente dos demais trabalhos que compararam um número maior de intervalos de idade, o presente estudo analisou somente dois períodos (até 220 e mais de 220 dias). Como cada granja foi analisada separadamente, não foi possível aumentar o número de tratamentos (faixas de idade) devido à limitação do número de repetições, tendo em vista que a maior concentração de inseminações ocorre por volta de 210 a 240 dias de idade.

Analisando as informações percebe-se que o intervalo de idade ideal para a primeira cobertura variou na literatura citada anteriormente. No entanto, para concluir os melhores resultados, estes autores levaram em conta a idade das fêmeas sem considerar o número de estros previamente ocorridos. De acordo com Brooks \& Smith (1980), os estudos que demonstram que o aumento da idade à primeira inseminação leva a um aumento do número de leitões nascidos são equivocados.

Para comprovar esta hipótese, Brooks \& Smith (1980), analisaram 64 marrãs mestiças de uma mesma granja, as quais foram inseminadas no segundo estro, mas em idades diferentes. Estes animais foram distribuídos em dois grupos. No primeiro, a média das idades em que as fêmeas foram inseminadas foi de 197,8 dias, sendo que no segundo foi de 237,2 dias. O número de nascidos totais e nascidos vivos não diferiram entre os grupos $(\mathrm{P}>0,05)$. Com base nestes dados, os autores concluíram que é mais relevante considerar o número de estros apresentados do que somente a idade da fêmea, para decidir o melhor momento da inseminação.

\section{Número de estros à primeira inseminação e desempenho reprodutivo no primeiro parto}

Analisando os efeitos do número de estros à primeira inseminação, não foram observadas diferenças no tamanho das leitegadas $(\mathrm{P}>0,05)$ nas sete granjas estudadas (Tabela 3). Foram avaliadas as inseminações ocorridas do segundo ao quinto estro. As inseminações realizadas no primeiro estro não foram tabuladas e analisadas, devido o reduzido número de repetições, conforme descrito na metodologia.

Tabela 3 - Efeito do número de estros à primeira inseminação (NEPI) no tamanho da leitegada do primeiro parto

\begin{tabular}{|c|c|c|c|c|c|c|}
\hline GRANJA & NEPI & $\mathrm{N}$ & $\mathrm{MM}$ & NM & $\mathrm{NV}$ & NT \\
\hline \multirow{4}{*}{ A } & & & $\begin{array}{ll}\mathrm{m} & \mathrm{s}\end{array}$ & $\mathrm{m} \quad \mathrm{s}$ & $\mathrm{m} \quad \mathrm{s}$ & $\mathrm{m}$ \\
\hline & 2 & 207 & $0,25 \pm 0,63$ & $0,42 \pm 0,79$ & $11,05 \pm 2,75$ & $11,72 \pm 2,75$ \\
\hline & 3 & 626 & $0,28 \pm 0,65$ & $0,44 \pm 0,82$ & $10,62 \pm 3,11$ & $11,34 \pm 3,11$ \\
\hline & 4 & 161 & $0,30 \pm 0,87$ & $0,33 \pm 0,72$ & $10,88 \pm 2,81$ & $11,51 \pm 2,69$ \\
\hline \multirow{3}{*}{ B } & 2 & 85 & $0,22 \pm 0,78$ & $0,43 \pm 0,70$ & $10,79 \pm 2,95$ & $11,45 \pm 2,99$ \\
\hline & 3 & 297 & $0,28 \pm 0,68$ & $0,48 \pm 0,92$ & $10,33 \pm 3,01$ & $11,09 \pm 3,05$ \\
\hline & 4 & 102 & $0,37 \pm 1,01$ & $0,47 \pm 0,68$ & $10,88 \pm 3,00$ & $11,72 \pm 3,10$ \\
\hline \multirow{2}{*}{$\mathrm{C}$} & 3 & 32 & $0,31 \pm 0,86$ & $0,56 \pm 1,29$ & $10,34 \pm 3,72$ & $11,22 \pm 3,65$ \\
\hline & 4 & 31 & $0,10 \pm 0,40$ & $0,22 \pm 0,42$ & $10,16 \pm 3,13$ & $10,48 \pm 3,02$ \\
\hline \multirow{2}{*}{$\mathrm{D}$} & 3 & 78 & $0,09 \pm 0,33$ & $0,26 \pm 0,59$ & $11,61 \pm 2,77$ & $11,96 \pm 2,87$ \\
\hline & 4 & 48 & $0,10 \pm 0,42$ & $0,33 \pm 0,66$ & $10,92 \pm 3,06$ & $11,35 \pm 3,16$ \\
\hline \multirow{2}{*}{$\mathrm{E}$} & 2 & 52 & $0,35 \pm 0,62$ & $0,56 \pm 0,89$ & $11,29 \pm 2,65$ & $12,19 \pm 2,75$ \\
\hline & 3 & 64 & $0,33 \pm 0,73$ & $0,45 \pm 0,73$ & $12,36 \pm 2,13$ & $13,14 \pm 2,35$ \\
\hline \multirow{3}{*}{$\mathrm{F}$} & 3 & 48 & $0,35 \pm 0,56$ & $0,52 \pm 0,99$ & $10,08 \pm 2,76$ & $10,79 \pm 3,13$ \\
\hline & 4 & 77 & $0,19 \pm 0,49$ & $0,31 \pm 0,67$ & $10,60 \pm 3,11$ & $11,26 \pm 3,46$ \\
\hline & 5 & 49 & $0,20 \pm 0,50$ & $0,43 \pm 0,68$ & $10,65 \pm 3,01$ & $11,47 \pm 3,28$ \\
\hline \multirow{2}{*}{$\mathrm{G}$} & 2 & 22 & $0,45 \pm 0,74$ & $0,32 \pm 0,48$ & $11,50 \pm 2,42$ & $12,18 \pm 2,48$ \\
\hline & 3 & 39 & $0,28 \pm 0,56$ & $0,31 \pm 0,57$ & $12,10 \pm 2,30$ & $12,82 \pm 2,67$ \\
\hline
\end{tabular}

Não houve diferença entre tratamentos na mesma granja $(\mathrm{P}>0,05)$ pelo teste $\mathrm{F}$, quanto ao tamanho da leitegada.

N: número de repetições; MM: mumificados; NM: natimortos; NV: nascidos vivos; NT: nascidos totais; m: média; s: desvio padrão. NEPI: número de estros à primeira inseminação.

Os valores do presente estudo corroboram com Young \& King (1981) os quais não verificaram diferença $(\mathrm{P}>0,05)$ no número de nascidos totais $\mathrm{e}$ nascidos vivos no primeiro parto entre as marrãs da raça Yorkshire, quando inseminadas no primeiro ou no terceiro estro. Também Young et al. (1990) não encontraram influência no número de nascidos, quando inseminaram animais Yorkshire $\mathrm{x}$ Landrace no primeiro, segundo ou no terceiro estro. Entretanto, vale ressaltar que, no presente experimento, foram avaliados os resultados obtidos de inseminações realizadas a partir do segundo estro, tendo em vista que o número de fêmeas inseminadas no primeiro foi insignificante. 
Semelhante ao presente estudo, Cottney et al. (2012) avaliaram em apenas uma granja, o tamanho das leitegadas nas inseminações ocorridas até o quinto estro observado. Também não foi observada influência do número do estro em que as marrãs foram inseminadas no número de leitões nascidos vivos, natimortos e nascidos totais $(\mathrm{P}>0,05)$. No entanto, neste estudo foi utilizado animais cruzados e que atingiram a puberdade em uma idade mais avançada, em média 209 dias. Com isso, as fêmeas inseminadas no primeiro estro, provavelmente já possuíam uma maturidade sexual suficiente para um adequado desenvolvimento dos leitões, não diferindo das inseminadas nos estros posteriores. Neste trabalho, os autores avaliaram o número de partos de toda a vida reprodutiva da marrã, o que não foi possível no presente estudo, tendo em vista que se tratava de uma linhagem mais recente, tendo então poucos partos por matriz. Os autores concluíram que as marrãs inseminadas no primeiro, segundo e terceiros estros completaram um maior número de partos e aquelas inseminadas no terceiro estro tiveram um maior número de leitões nascidos vivos durante a vida útil $(\mathrm{P}<0,01)$. Os autores identificaram que coberturas após o terceiro estro, não só aumentaram os custos de alimentação e os DNP, mas também reduziram o número de partos que a porca completou.

Em contrapartida, nos estudos de Macpherson et al. (1977), com fêmeas mestiças e Kummer et al. (2005), com fêmeas da linhagem Camborough ${ }^{\circledR} 22$, o número de nascidos totais e nascidos vivos no primeiro parto de marrãs inseminadas no primeiro estro foram menores $(\mathrm{P}<0,05)$ em relação aquelas fêmeas inseminadas no segundo, terceiro e quarto estro. Apesar do número de animais inseminados no primeiro estro não ter sido analisado no presente estudo, é conhecido que o estro puberal é muito variável na sua duração e no número médio de ovulações, por isso não é recomendado para a cobertura (VAN DER LENDE \& SCHOENMAKER, 1990). Além disso, Cottney et al. (2012) verificaram um maior número de leitões natimortos, demonstrando a baixa eficiência das inseminações realizadas neste estro.

\section{CONCLUSÕES}

A partir desse estudo, pode-se concluir para a linhagem Camborough ${ }^{\circledR} 25$ que o tamanho da leitegada no primeiro parto não é influenciado pela idade, quando inseminadas a partir de 190 dias. O tamanho da leitegada no primeiro parto também não é influenciado pela idade em que ocorre o primeiro estro da fêmea.

Conclui-se também que, o segundo estro da marrã pode ser utilizado sem que ocorra comprometimento na taxa de repetição de estro e no total de nascidos.

Diante destas considerações, as marrãs devem ser inseminadas já a partir do segundo estro, visando um menor período de dias não produtivos, sem comprometimento da eficiência reprodutiva.

\section{REFERÊNCIAS}

Associação Brasileira da Indústria Produtora e Exportadora de Carne Suína. Disponível em: <http://www.abipecs.org.br>. Acesso em: 27/11/2012.

BABOT, D.; CHAVEZ, E. R.; NOGUERA, J. L. The effect of age at the first mating and herd size on the lifetime productivity of sows. Animal Research, v.52, p.49-64, 2003.

BROOKS, P. H., SMITH, D. A. The effect of mating age on the reproductive performance, food utilization and live weight change of the female pig. Livestock Production Science, v.7, p.67-78, 1980.

CLARK, L. K.; LEMAN, A. D.; MORRIS, R. Factors that influence litter size in swine: parity one females. Journal of the American Veterinary Medical Association, v.192, n.2, p.187-194, 1988.

COTTNEY, P. D.; MAGOWAN, E.; BALL, M. E. E.; GORDON, E. Effect of oestrus number of nulliparous sows at first service on first litter and lifetime performance. Livestock Science, v.146, p.5-12, 2012.

KARLBOM, I.; EINARSSON, S.; EDQVIST, L. E. Attainment of puberty in female pigs: clinical appearance and patterns of progesterone, oestradiol$17 \beta$ and LH. Animal Reproduction Science, v.4, p.301-312, 1982.

KIRKWOOD, R. N.; AHERNE, F.X. Energy intake, body composition and reproductive performance of the gilt. Journal of Animal Science, v.60, p.1518-29, 1985.

KUMMER, R.; BORTOLOZZO, F. P.; WENTZ, I.; BERNARDI, M. L. Existe diferença no desempenho reprodutivo ao primeiro parto de leitoas inseminadas no $1^{\circ}, 2^{\circ}, 3^{\circ}$ ou $4^{\circ}$ estro? Acta ScientiaeVeterinariae, v.33, n.2, p.125-130, 2005.

LUCIA Jr, T.; DIAL, G. G.; MARSH, W. E. Lifetime reproductive performance in female pigs having distinct reasons for removal. Livestock Production Science, v.63, p.213-222, 2000.

LUCIA Jr., T.; BIANCHI, I.; RECH, H.; CORREA, M. N.; DESCHAMPS, J. C. Parâmetros de desempenho reprodutivo de fêmeas nulíparase primíparas em função da idade ao primeiro serviço,Goiânia, GO, 2003. In: $11^{\circ}$ CONGRESSO BRASILEIRO DE VETERINÁRIOS ESPECIALISTAS EM SUÍNOS, 2003, Anais... ABRAVES, 2003, v.1, p.175-176.

MACPHERSON, R. M.; HOVELL, F. D. D.; JONES, A. S. Performance of sows first mated at puberty or second or third oestrus, and carcass assessment of oncebred gilts. Animal Production, v.24, p.333-342, 1977.

MARTIN RILLO, S.; DE ALBA, C.; FALCETO, M. V.; PERALTA, W.; BUSTAMANTE, J. Efectodel aparato genital de laprimeriza sobre laproductividad de 
la cerda, 2000. Articulos Porcino Archivo. Disponível em:<http://albeitar.portalveterinaria.com/noticia/3511>. Acesso em: 12/01/2013.

PATERSON, A. M. The controlled induction of puberty In: COLE, D.J.A.; FOXCROFT, G.R. Control of Pig Reproduction, p.139-159, 1982.

PATTERSON, J. L.; BELTRANENA, E.; FOXCROFT, G. R. The effect of gilt age at first estrus and breeding on third estrus on sow body weight changes and long-term reproductive performance. Journal Animal Science, v.88, p.2500-2513, 2010.

ROONGSITTHICHAI, A.; CHEUCHUCHART, P.; CHATWIJITKUL, S.; CHANTAROTHAI, O. Influence of age first estrus, body weight, and average daily gain of replacement gilts on their subsequent reproductive performance as sows. Livestock Science, v.151, p.238245, 2013.

SAITO, H.; SASAKI, Y.; KOKETSU, Y. Associations between age of gilts at first mating and lifetime performance or culling risk in commercial herds. Journal Veterinary Medical Science, v.73, p.555-559, 2011.

SAMPAIO, I. B. M. Estatística aplicada à experimentação animal. Belo Horizonte: Fundação de Ensino e Pesquisa em Medicina Veterinária e Zootecnia, 2002, 265p.
SCHUKKEN, Y. H.; BUURMAN, J.; HUIRNE, R. B. M.; WILLEMSE, A. H.; VERNOOY, J. C. M.; VAN DER BROEK, J.; VERHEIJDEN, J. H. M. Evaluation of optimal age at first conception in gilts from data collected in commercial swine herds. Journal of Animal Science, v.72, p.1387-1392, 1994.

SISTEMA de análise estatística e genética (SAEG), UFV, Central de processamento de dados, Viçosa- MG., 1999.

VAN DER LENDE, T.; SCHOENMAKER, G. J. N. The relationship between ovulation rate and litter size before and after day 35 of pregnancy in gilts mid sows: an analysis of published data. Livestock Production Science, v.26, p.217-229, 1990.

YOUNG, L. G.; KING, G. J. Reproductive performance of gilts bred on first versus third estrus. Journal of Animal Science, v.53, n.1, p.19-25, 1981.

YOUNG, L. G.; KING, G. J; WALTON, J. S.; McMILLAN, I.; KLEVORICK, M. Age, weight, backfat and time of mating effects on performance of gilts. Canadian Journal of Animal Science, v.70, p.469-481, 1990. 